International Journal of Pure and Applied Mathematics

Volume 109 No. 4 2016, 869-879

ISSN: 1311-8080 (printed version); ISSN: 1314-3395 (on-line version)

url: http://www.ijpam.eu

doi: 10.12732 /ijpam.v109i4.10

ijpam.eu

\title{
ON GRADED 2-ABSORBING PRIMARY SUBMODULES
}

\author{
Ece Yetkin Celikel \\ Department of Mathematics \\ Faculty of Art and Science \\ Gaziantep University \\ Gaziantep, TURKEY
}

\begin{abstract}
In this paper, we introduce and study the concept of graded 2-absorbing primary submodules of graded modules over graded commutative rings generalizing graded 2-absorbing submodules. Let $R$ be a graded ring and $M$ be a graded $R$-module. A proper graded submodule $N$ of $M$ is called a graded 2-absorbing primary submodule of $M$ if whenever $a, b \in h(R)$ and $m \in h(M)$ and $a b m \in N$, then $a m \in M-\operatorname{Gr}(N)$ or $b m \in M-\operatorname{Gr}(N)$ or $a b \in\left(N:_{R} M\right)$.
\end{abstract}

AMS Subject Classification: $13 \mathrm{~A} 02$

Key Words: graded 2-absorbing ideal, graded 2-absorbing primary ideal, graded 2-absorbing submodule, graded 2-absorbing primary submodule

\section{Introduction and Preliminaries}

Let $G$ be a multiplicative group with identity $e$ and $R$ be a commutative ring with $1 \neq 0 . R$ is called a $G$-graded ring if there exist additive subgroups $R_{g}$ of $R$ indexed by the elements $g \in G$ such that $R=\oplus_{g \in G} R_{g}$ and $R_{g} R_{h} \subseteq R_{g h}$ for all $g, h \in G$. The elements of $R_{g}$ are called homogeneous of degree $g$ and the set of all the homogeneous elements are denoted by $h(R)$, i.e. $h(R)=\cup_{g \in G} R_{g}$. If $a \in R$, then the element $a$ can be written uniquely as $\sum_{g \in G} a_{g}$, where $a_{g}$ is called the $g$-component of $a$ in $R_{g}$. In this case, $R_{e}$ is a subring of $R$ and $1_{R} \in R_{e}$. Let $R$ be a $G$-graded ring and $M$ an $R$-module. We call $M$ as a graded $R$-module if there exists a family of subgroups $\left\{M_{g}\right\}_{g \in G}$ of $M$ such that $M=\oplus_{g \in G} M_{g}$ and $R_{g} M_{h} \subseteq M_{g h}$ for all $g, h \in G$ where $R_{g} M_{h}$ denotes the additive subgroup of $M$

$\begin{array}{lcc}\text { Received: } & \text { July 18, } 2016 & \text { C } 2016 \text { Academic Publications, Ltd. } \\ \text { Revised: } & \text { August 25, } 2016 & \text { url: www.acadpubl.eu } \\ \text { Published: } & \text { October } 7,2016 & \end{array}$


consisting of all finite sums of elements $r_{g} s_{h}$ with $r_{g} \in R_{g}$ and $s_{h} \in M_{h}$. Also, we write $h(M)=\cup_{g \in G} M_{g}$ and the elements of $h(M)$ are called homogeneous. Let $M=\oplus_{g \in G} M_{g}$ is a graded $R$-module, then the subgroup $M_{g}$ of $M$ is an $R_{e}$ -module for all $g \in G$. Let $N$ be a submodule of $M$. Then $N$ is called a graded submodule of $M$ if $N=\oplus_{g \in G} N_{g}$ where $N_{g}=N \cap M_{g}$ for $g \in G$. In this case, $N_{g}$ is called the $g$-component of $N$. Moreover, $M / N$ becomes a graded $R$-module with $g$-component $(M / N)_{g}=\left(M_{g}+N\right) / N$ for $g \in G$ [11]. The graded radical of $I$ (in abbreviation $\operatorname{Gr}(I)$ ) is the set of all $x \in R$ such that for each $g \in G$ there exists $n_{g}>0$ with $x_{g}^{n_{g}} \in I$. Note that, if $r$ is a homogeneous element of $G(R)$, then $r \in G r(I)$ if and only if $r^{n} \in I$ for some $n \in N$ [13]. Let $R$ be a graded ring, $M$ a graded $R$-module and $N$ a graded submodule of $M$. We will denote by $\left(N:_{R} M\right)$ the residual of $N$ by $M$, that is, the set of all $r \in h(R)$ such that $r M \subseteq N$. Graded multiplication modules are introduced by S.E Atani and R.E Atani in [7]: A graded $R$-module $M$ is said to be a graded multiplication module if for each graded submodule $N$ of $M, N=I M$ for some graded ideal $I$ of $R$. We say that $I$ is a presentation graded ideal of $N$. Note that since $I \subseteq\left(N:_{R} M\right)$, then $N=I M \subseteq\left(N:_{R} M\right) M \subseteq N$. So $N=\left(N:_{R} M\right) M$. Let $N=I M$ and $K=J M$ be graded submodules of a graded multiplication $R$-module $M$. Then the product of $N$ and $K$ is independent from their graded presentation ideals $I$ and $K$. [12] It can be easily understood that if a graded module is multiplication, then it is a graded multiplication module. However the converse of this observation is not true. There is an example in [14] for a graded multiplication ring which is not multiplication. The group $\operatorname{ring} R[Z]$, where $R$ is a Dedekind domain is a graded Dedekind domain and so it is a graded multiplication domain. On the other hand, if $R$ is not a field, then $R[Z]$ is not a Dedekind domain and so it is not a multiplication domain. Thus a graded multiplication module need not be multiplication module. Let $N$ be a proper graded submodule of a nonzero graded $R$-module $M$. Then the $M$ graded radical of $N$, denoted by $M-G r(N)$, is defined to be the intersection of all graded prime submodules of $M$ containing $N$. If $M$ has no graded prime submodule containing $N$, then we say $M-G r(N)=M$. It is shown in $[12$, Theorem 9] that if $N$ is a proper graded submodule of a graded multiplication $R$-module $M$, then $M-G r(N)=G r\left(\left(N:_{R} M\right)\right) M$.

Let $R$ be a graded commutative ring with $1 \neq 0$ and $M$ be a graded $R$ module. Graded prime and graded primary ideals have been introduced and studied in [13]. A proper graded ideal $P$ of $R$ is said to be graded prime (resp. graded weakly prime) ideal if whenever $a, b \in h(R)$ with $a b \in P$ (resp. $0 \neq$ $a b \in P$ ), then either $a \in P$ or $b \in P$ [13]. A proper graded ideal $I$ of $R$ is said to be a graded primary ideal of $R$ if whenever $a, b \in h(R)$ with $a b \in I$, then 
$a \in I$ or $a \in G r(I)$. [13]. Graded prime and graded primary submodules of $M$ have been studied in [3], [4], [5], [12]. A proper graded submodule $N$ of $M$ is said to be graded prime (resp. graded weakly prime) submodule if whenever $a \in h(R)$ and $m \in h(M)$ with $a m \in N$ (resp. $0 \neq a m \in N$ ), then either $a \in(N: R M)$ or $m \in N$. A proper graded ideal $I$ of $R$ is said to be graded 2-absorbing (graded weakly 2-absorbing) ideal if whenever $a, b, c \in h(R)$ with $a b c \in I$ (resp. $0 \neq a b c \in I$ ), then either $a b \in I$ or $b c \in I$ or $a c \in I$. Recently, graded 2-absorbing and weakly graded 2-absorbing submodules of $M$ have been introduced in [1]. A proper graded submodule $N$ of $M$ is said to be graded 2absorbing (graded weakly 2-absorbing) submodule if whenever $a, b \in h(R)$ and $m \in h(M)$ with $a b m \in N$ (resp. $0 \neq a b m \in N)$ then either $a m \in N$ or $b m \in N$ or $a b \in(N: R M)$. As it is defined in [15], a graded ideal $I$ of $R$ is said to be a graded 2-absorbing primary ideal if whenever $a, b, c \in h(R)$ with $a b c \in I$, then either $a b \in I$ or $b c \in G r(I)$ or $a c \in G r(I)$. In this paper we intoduce the concept of graded 2-absorbing primary submodules as follows: Let $R$ be a $G$-graded ring, $N$ be a proper graded submodule of a graded $R$-module $M$ and $g \in G$. We say a proper $R_{e}$-submodule $N_{g}$ is to be a g-2-absorbing primary submodule of $M_{g}$ if whenever $a, b \in R_{e}$ and $m \in M_{g}$ with $a b m \in N_{g}$, then $a b \in\left(N_{g}: R_{e} M_{g}\right)$ or am $\in M_{g}-\operatorname{Gr}\left(N_{g}\right)$ or $b m \in M_{g}-\operatorname{Gr}\left(N_{g}\right)$. We say $N$ to be a graded 2-absorbing primary submodule of $M$ if whenever $a, b \in h(R)$ and $m \in h(M)$ with abm $\in N$, then $a b \in\left(N:_{R} M\right)$ or $a m \in M-G r(N)$ or $b m \in M-G r(N)$. We give some basic results of this class of graded submodules and discuss on the relations among graded 2-absorbing ideals, graded 2-absorbing primary ideals, graded 2-absorbing submodules and graded 2-absorbing primary submodules.

\section{Properties of Graded 2-Absorbing Primary Submodules}

Lemma 1. Let $R$ be a graded ring and $M$ be a graded $R$-module. If $N$ is a graded 2-absorbing primary submodule of $M$, then $N_{g}$ is a $g$-2-absorbing primary $R_{e}$-submodule of $M_{g}$ for all $g \in G$.

Proof. Let $a, b \in R_{e}, m \in M_{g}$ with $a b m \in N_{g}$. Since $N$ is a graded 2absorbing primary submodule of $M$ and $N_{g}=\left(N \cap M_{g}\right) \subset N$ we get either $a b \in\left(N:_{R} M\right)$ or $a m \in M-G r(N)$ or $b m \in M-G r(N)$. If $a b \in\left(N:_{R} M\right)$, then $a b \in\left(N_{g}:_{R_{e}} M_{g}\right)$ as $\left(N:_{R} M\right) \subset\left(\left(N \cap M_{g}\right):_{R_{e}} M_{g}\right)=\left(N_{g}:_{R_{e}} M_{g}\right)$. Suppose that $a m \in M-G r(N)$. Since $a m \in M_{g}$ and $a m \in M-G r(N)$, we have $a m \in M$ $G r(N) \cap M-\operatorname{Gr}\left(M_{g}\right)=M-\operatorname{Gr}\left(N \cap M_{g}\right)=M-\operatorname{Gr}\left(N_{g}\right)$. If $b m \in M-G r(N)$, then similarly we conclude that $b m \in M-\operatorname{Gr}\left(N_{g}\right)$. Thus $N_{g}$ is a $g$-2-absorbing primary $R_{e}$-submodule of $M_{g}$. 
We need the following Lemma as it is used in the most of the proofs in this paper.

Lemma 2. [6], [7], [12] Let $M$ be a graded module over a G-graded ring $R$. Then

1. If $N$ is a graded submodule of $M, I$ is a graded ideal of $R, a \in h(R)$ and $m \in h(M)$, then $R m, I N$ and $a N$ are graded submodules of $M$, and $R a$, $(N: R M)$ are graded ideals of $R$.

2. If $\left\{N_{i}\right\}_{i \in \Lambda}$ is a collection of graded submodules of $M$, then $\sum_{i \in \Lambda} N_{i}$ and $\bigcap_{i \in \Lambda} N_{i}$ are graded submodules of $M$.

3. $M$ is a graded multiplication $R$-module if and only if for each $m$ in $h(M)$ there exists a graded ideal $I$ of $R$ such that $R m=I M$.

4. Every homomorphic image of a graded multiplication module is a graded multiplication module.

Theorem 3. Let $M$ be a finitely generated graded multiplication $R$ module, and $N$ be a graded 2-absorbing primary submodule of $M$. Then the following statements hold.

1. If $a b K \subseteq N$ where $a, b \in R$ and $K$ is a $R$-submodule of $M$, then $a b \in$ $(N: R M)$ or $a K \subseteq M-G r(N)$ or $b K \subseteq M-G r(N)$.

2. If $a b K \subseteq N_{g}$ where $a, b \in R_{e}$ and $K$ is a $R_{e}$-submodule of $M_{g}$, then $a b \in\left(N_{g}:_{R_{e}} M_{g}\right)$ or $a K \subseteq M-\operatorname{Gr}\left(N_{g}\right)$ or $b K \subseteq M-\operatorname{Gr}\left(N_{g}\right)$.

3. $\left(N_{g}:_{R_{e}} M_{g}\right)$ is a $g$-2-absorbing primary ideal of $R_{e}$.

Proof. (1) Assume that $a b K \subseteq N$ but $a b \notin\left(N:_{R} M\right), a K \nsubseteq M-G r(N)$ and $b K \nsubseteq M-G r(N)$. Then $a k_{1} \notin M-G r(N)$ and $b k_{2} \notin M-G r(N)$ for some $k_{1}, k_{2} \in h(K)$. Since $a b k_{1} \in N$ and $a b \notin\left(N:_{R} M\right)$ and $a k_{1} \notin M-G r(N)$, we have $b k_{1} \in M-G r(N)$. Since $a b k_{2} \in N$ and $a b \notin\left(N:_{R} M\right)$ and $b k_{2} \notin M-G r(N)$, we have $a k_{2} \in M-G r(N)$. Now, since $a b\left(k_{1}+k_{2}\right) \in N$ and $a b \notin\left(N:_{R} M\right)$, we have $a\left(k_{1}+k_{2}\right) \in M-G r(N)$ or $b\left(k_{1}+k_{2}\right) \in M-G r(N)$. Suppose that $a\left(k_{1}+k_{2}\right)=a k_{1}+a k_{2} \in M-G r(N)$. Since $a k_{2} \in M-G r(N)$, we have $a k_{1} \in M-$ $G r(N)$, a contradiction. Suppose that $b\left(k_{1}+k_{2}\right)=b k_{1}+b k_{2} \in M-G r(N)$. Since $b k_{1} \in M-G r(N)$, we have $b k_{2} \in M-G r(N)$, a contradiction again. Thus $a K \subseteq M-G r(N)$ or $b K \subseteq M-G r(N)$. 
(2) Suppose that $N$ is a graded 2-absorbing primary submodule of $M$. Then $N_{g}$ is a $g$-2-absorbing primary $R_{e}$-submodule of $M_{g}$ by Lemma 1 . So we are done from part (1).

(3) Let $a, b, c \in R_{e}$ such that $a b c \in\left(N_{g}: R_{e} M_{g}\right), a c \notin G r\left(N_{g}: R_{e} M_{g}\right)$ and $b c \notin G r\left(N_{g}: R_{e} M_{g}\right)$. Put $K=c M_{g}$. Then $K$ is a $R_{e}$-submodule of $M_{g}$. Since $N_{g}$ is a $g$-2-absorbing primary submodule of $M_{g}$ and $a b K \subseteq N_{g}$, we have that either $a b \in\left(N_{g}:_{R_{e}} M_{g}\right)$ or $a K \subseteq M-\operatorname{Gr}\left(N_{g}\right)$ or $b K \subseteq M-\operatorname{Gr}\left(N_{g}\right)$ by (1). Since $a c \notin G r\left(N_{g}:_{R_{e}} M_{g}\right)$ and $b c \notin G r\left(N_{g}:_{R_{e}} M_{g}\right)$, we conclude that $a K \nsubseteq \operatorname{Gr}\left(\left(N_{g}:_{R_{e}} M_{g}\right)\right) M=M-\operatorname{Gr}\left(N_{g}\right)$ and $b K \nsubseteq \operatorname{Gr}\left(\left(N_{g}:_{R_{e}} M_{g}\right)\right) M=$ $M-\operatorname{Gr}\left(N_{g}\right)$. Thus $a b \in\left(N_{g}: R_{e} M_{g}\right)$, which shows that $\left(N_{g}: R_{e} M_{g}\right)$ is a $g$-2-absorbing primary ideal of $R_{e}$.

Recall that a graded $R$-module $M$ is caled graded cyclic if $M=R m$ where $m \in h(M)$. It is well-known that $M$ is a graded cyclic module, then $M$ is a graded multiplication module.

Theorem 4. (1) Let $M_{g}$ be a cyclic $R_{e}$-submodule and $N$ a graded submodule of $M_{g}$. Then $N_{g}$ is a $g$-2-absorbing primary $R_{e}$-submodule of $M_{g}$ if and only if $\left(N_{g}:_{R_{e}} M_{g}\right)$ is a $g$-2-absorbing primary ideal of $R_{e}$.

(2) If $M$ is a cyclic $R$-module and $N$ a graded submodule of $M$, then $N$ is a graded 2-absorbing primary submodule of $M$ if and only if $\left(N:_{R} M\right)$ is a graded 2-absorbing primary ideal of $R$.

Proof. (1) If $N_{g}$ is a $g$-2-absorbing primary $R_{e}$-submodule of $M_{g}$, then $\left(N_{g}: R_{e} M_{g}\right)$ is a $g$-2-absorbing primary ideal of $R_{e}$ by Theorem 3 . Conversely, let $a, b \in R_{e}, m \in M_{g}$ such that $a b m \in N_{g}$. Since $M_{g}$ is a graded cyclic $R_{e}$-module, $M_{g}=R_{e} x$ for some $x \in M$. Then there is $c \in R_{e}$ such that $m=c x$. Since $a b c \in\left(N_{g}:_{R_{e}} M_{g}\right)$ and $\left(N_{g}:_{R_{e}} M_{g}\right)$ is a $g$-2-absorbing primary ideal of $R_{e}$, we get either $a b \in\left(N_{g}:_{R_{e}} M_{g}\right)$ or $a c \in G r\left(N_{g}:_{R_{e}} M_{g}\right)$ or $b c \in \operatorname{Gr}\left(N_{g}: R_{e} M_{g}\right)$. If $a b \in\left(N_{g}: R_{e} M_{g}\right)$, then we are done. So suppose that $a c \in G r\left(N_{g}:_{R_{e}} M_{g}\right)$. Thus $a m=a c x \in G r\left(N_{g}:_{R_{e}} M_{g}\right) M_{g}=M_{g}-\operatorname{Gr}\left(N_{g} \dot{j}\right.$. Similary if $b c \in \operatorname{Gr}\left(N_{g}:_{R_{e}} M_{g}\right)$, then we have $b m \in M_{g}-\operatorname{Gr}\left(N_{g}\right)$, so we are done.

(2) One can easily verify similar to part (1).

Theorem 5. Let $M$ be a graded $R$-module and $N$ be a graded submodule of $M$. If $M-G r(N)$ is a graded prime submodule of $M$, then $N$ is a graded 2-absorbing primary submodule of $M$.

Proof. Suppose that $M-G r(N)$ is a graded prime submodule of $M$ and $a b m \in N, a m \notin M-G r(N)$ for some $a, b \in h(R)$ and $m \in h(M)$. Since $M$ $\operatorname{Gr}(N)$ is a graded prime submodule and $a b m \in M-G r(N)$, then $b \in(M-$ 
$\left.G r(N):_{R} M\right)$. So $b m \in M-G r(N)$. Consequently $N$ is a graded 2-absorbing primary submodule of $M$.

We say that a graded $R$-module $M$ is a graded divided module if for every graded prime submodule $N$ of $M$, we have $N \subseteq R m$ for all $m \in M \backslash N$.

Theorem 6. Let $R$ be a graded ring. If $M$ is a divided graded $R$-module, then every proper submodule of $M$ is a graded 2-absorbing primary submodule of $M$. In particular, every proper graded submodule of a graded chained module is a graded 2-absorbing primary submodule.

Proof. Let $N$ be a proper graded submodule of $M$. Since the graded prime submodules of a graded divided module are linearly ordered, we conclude that $M-G r(N)$ is a graded prime submodule of $M$. Hence $N$ is a graded 2-absorbing primary submodule of $M$ by Theorem 5 .

Lemma 7. Let $R$ be a graded ring and $I$ be a proper graded ideal of $R$. Then $I$ is a graded 2-absorbing primary ideal if and only if whenever $I_{1} I_{2} I_{3} \subseteq I$ for some ideals $I_{1}, I_{2}, I_{3}$ of $R$, then $I_{1} I_{2} \subseteq I$ or $I_{2} I_{3} \subseteq G r(I)$ or $I_{1} I_{3} \subseteq G r(I)$.

Proof. It can be easily obtained by the similar argument in the proof of Theorem 2.19 in [8].

Now we present the general form of Theorem 4 just under the condition that $M$ is a graded multiplication.

Theorem 8. Let $M$ be a graded multiplication module over graded ring $R$ and $N$ be a graded submodule of $M$. If $\left(N:_{R} M\right)$ is a graded 2-absorbing primary ideal of $R$, then $N$ is a graded 2-absorbing primary submodule of $M$.

Proof. Suppose that $\left(N:_{R} M\right)$ is a graded 2-absorbing primary ideal of $R$ and $I_{1} I_{2} K \subseteq N$ for some graded ideals $I_{1}, I_{2}$ of $R$ and some graded

submodule $K$ of $M$ and $I_{1} I_{2} \nsubseteq\left(N:_{R} M\right)$. Here, there exists a graded ideal $I_{3}$ of $R$ with $K=I_{3} M$ as $M$ is graded multiplication. Then $I_{1} I_{2} I_{3} \subseteq$ $\left.\chi N:_{R} M\right)$. Since $\left(N:_{R} M\right)$ is graded 2-absorbing primary, we conclude that either $I_{2} I_{3} \subseteq G r\left(N:_{R} M\right)$ or $I_{1} I_{3} \subseteq G r\left(N:_{R} M\right)$ by Lemma 7. Without loss generality assume that $I_{2} I_{3} \subseteq G r\left(N:_{R} M\right)$. Therefore $I_{2} I_{3} M=I_{2} K \subseteq$ $\left(G r\left(N:_{R} M\right)\right) M=M-G r(N)$, we are done.

Note that the restriction on $M$ is necessary in Theorem 8 as one can see by the following example. 
Example 9. Let $R=\mathbb{Z}=R_{0}$ as $\mathbb{Z}$-graded ring and $M=\mathbb{Z} \times \mathbb{Z} \times \mathbb{Z}$ as $\mathbb{Z}$-graded $R$-module with $M_{0}=\{0\} \times \mathbb{Z} \times \mathbb{Z}, M_{2}=\mathbb{Z} \times\{0\} \times \mathbb{Z}$ and $M_{3}=$ $\mathbb{Z} \times \mathbb{Z} \times\{0\}$. Now consider a graded submodule $N=2 \mathbb{Z} \times 3 \mathbb{Z} \times\{0\}$. Observe that $(N: R M)=0$ is a graded 2-absorbing primary ideal of $R$. However $N$ is not a graded 2-absorbing primary submodule of $M$. To see this, first observe that $2 \mathbb{Z} \times \mathbb{Z} \times \mathbb{Z}, \mathbb{Z} \times 3 \mathbb{Z} \times \mathbb{Z}, \mathbb{Z} \times \mathbb{Z} \times\{0\}$ are graded prime submodules of $M$ containing $N$. Then $M-G r(N) \subseteq 2 \mathbb{Z} \times \mathbb{Z} \times \mathbb{Z} \cap \mathbb{Z} \times 3 \mathbb{Z} \times \mathbb{Z} \cap \mathbb{Z} \times \mathbb{Z} \times\{0\}=N$. Since 2.3. $(1,1,0) \in N$ but neither $2.3 \in\left(N:_{R} M\right)$ nor 2.(1,1,0) $\in M-G r(N)$ nor 3.(1,1,0) $\in M-G r(N), N$ is not graded 2-absorbing primary submodule of $M$.

Theorem 10. Let $M$ be a graded multiplication $R$-module and $N_{1}$ and $N_{2}$ be graded primary submodules of $M$. Then $N_{1} \cap N_{2}$ is a graded 2-absorbing primary submodule of $M$.

Proof. Suppose that $N_{1}$ and $N_{2}$ are graded primary submodules of $M$. Then $\left(N_{1}:_{R} M\right)$ and $\left(N_{2}:_{R} M\right)$ are graded primary ideals of $R$ by Proposition 2.5 in [6]. Hence $\left(N_{1}:_{R} M\right) \cap\left(N_{2}:_{R} M\right)=\left(N_{1} \cap N_{2}:_{R} M\right)$ is a graded 2-absorbing primary ideal of $R$ by Theorem 2 in [15]. Thus $N_{1} \cap N_{2}$ is a graded 2-absorbing primary submodule of $M$ by Theorem 4 .

Theorem 11. Let $M$ be a graded multiplication $R$-module and $N_{1}, N_{2}, \ldots$, $N_{n}$ be graded 2-absorbing primary submodules of $M$ with the same $M$-graded radical. Then $N=\cap_{i=1}^{n} N_{i}$ is a graded 2-absorbing primary submodule of $M$.

Proof. Suppose that $a b m \in N$ for some $a, b \in h(R)$ and $m \in h(M)$ and $a b \notin\left(N:_{R} M\right)$. Then $a b \notin\left(N_{i}:_{R} M\right)$ for some $1 \leq i \leq n$. Hence $a m \in M$ $\operatorname{Gr}\left(N_{i}\right)=\cap_{i=1}^{n} M-\operatorname{Gr}\left(N_{i}\right)=M-G r(N)$ or $b m \in M-\operatorname{Gr}\left(N_{i}\right)=\cap_{i=1}^{n} M-\operatorname{Gr}\left(N_{i}\right)=$ $M-G r(N)$.

Theorem 12. Let $R$ be a graded ring and $N$ be a proper graded submodule of a graded $R$-module $M$. Then $N$ is graded 2-absorbing primary if and only if $\left(N:_{M} a b\right) \subseteq\left(M-G r(N):_{M} a\right)$ or $\left(N:_{M} a b\right) \subseteq\left(M-G r(N):_{M} b\right)$ for all $a, b \in h(R)$ with $a b \notin(N: R M)$.

Proof. Suppose that $N$ is a graded 2-absorbing primary submodule of $M$, $a b \notin\left(N:_{R} M\right)$ for some $a, b \in h(R)$ and $m \in\left(N:_{M} a b\right)$. Hence $a b m \in N$, which implies either $a m \in M-G r(N)$ or $b m \in M-G r(N)$. This means that $m \in\left(M-G r(N):_{M} a\right)$ or $m \in\left(M-G r(N):_{M} b\right)$, so we are done. The converse part is clear. 
Theorem 13. Let $R$ be a graded ring and $N$ be a proper graded submodule of a graded $R$-module $M$. Then $N$ is a graded 2-absorbing primary submodule of $M$ if and only if whenever $I_{1} I_{2} K \subseteq N$ for some graded ideals $I_{1}, I_{2}$ of $R$ and some graded submodule $K$ of $M$, then $I_{1} I_{2} \subseteq\left(N:_{R} M\right)$ or $I_{1} K \subseteq M-G r(N)$ or $I_{2} K \subseteq M-G r(N)$.

Proof. Suppose that $N$ is a graded 2-absorbing primary submodule of $M$ and $I_{1} I_{2} K \subseteq N$ for some graded ideals $I_{1}, I_{2}$ of $R$ and some graded submodule $K$ of $M$ and $I_{1} I_{2} \not \subseteq\left(N:_{R} M\right)$. We show that $I_{1} K \subseteq M-G r(N)$ or $I_{2} K \subseteq M$ $G r(N)$. Assume on the contrary that $I_{1} K \nsubseteq M-G r(N)$ and $I_{2} K \nsubseteq M-G r(N)$. Then $a_{1} K \nsubseteq M-G r(N)$ and $a_{2} K \nsubseteq M-G r(N)$ for some $a_{1} \in I_{1}$ and $a_{2} \in I_{2}$. Since $a_{1} a_{2} K \subseteq N$ and neither $a_{1} K \subseteq M-G r(N)$ nor $a_{2} K \subseteq M-G r(N)$, we have $a_{1} a_{2} \in(N: R M)$ by Theorem 3 .

Since $I_{1} I_{2} \nsubseteq\left(N:_{R} M\right)$, we have $b_{1} b_{2} \notin\left(N:_{R} M\right)$ for some $b_{1} \in I_{1}$ and $b_{2} \in I_{2}$. Since $b_{1} b_{2} K \subseteq N$ and $b_{1} b_{2} \notin\left(N:_{R} M\right)$, we have $b_{1} K \subseteq M-G r(N)$ or $b_{2} K \subseteq M-G r(N)$ by Theorem 3 . We consider three cases.

Case 1. Suppose that $b_{1} K \subseteq M-G r(N)$ but $b_{2} K \nsubseteq M-G r(N)$. Since $a_{1} b_{2} K \subseteq N$ and neither $b_{2} K \subseteq M-G r(N)$ nor $a_{1} K \subseteq M-G r(N)$, we conclude that $a_{1} b_{2} \in\left(N:_{R} M\right)$ by Theorem 3. Since $b_{1} K \subseteq M-G r(N)$ but $a_{1} K \nsubseteq M$ $G r(N)$, we conclude that $\left(a_{1}+b_{1}\right) K \nsubseteq M-G r(N)$. Since $\left(a_{1}+b_{1}\right) b_{2} K \subseteq N$ and neither $b_{2} K \subseteq M-G r(N)$ nor $\left(a_{1}+b_{1}\right) K \subseteq M-G r(N)$, we conclude that $\left(a_{1}+b_{1}\right) b_{2} \in\left(N:_{R} M\right)$ by Theorem 3. Since $\left(a_{1}+b_{1}\right) b_{2}=a_{1} b_{2}+b_{1} b_{2} \in\left(N:_{R}\right.$ $M)$ and $a_{1} b_{2} \in\left(N:_{R} M\right)$, we conclude $b_{1} b_{2} \in\left(N:_{R} M\right)$, a contradiction.

Case 2. Suppose that $b_{2} K \subseteq M-G r(N)$ but $b_{1} K \nsubseteq M-G r(N)$. Similar to the previous case we reach a contradiction.

Case 3. Suppose that $b_{1} K \subseteq M-G r(N)$ and $b_{2} K \subseteq M-G r(N)$. Since $b_{2} K \subseteq M-G r(N)$ and $a_{2} K \nsubseteq M-G r(N)$, we conclude that $\left(a_{2}+b_{2}\right) K \nsubseteq M$ $G r(N)$. Since $a_{1}\left(a_{2}+b_{2}\right) K \subseteq N$ and neither $a_{1} K \subseteq M-G r(N)$ nor $\left(a_{2}+b_{2}\right) K \subseteq$ $M-G r(N)$, we conclude that $a_{1}\left(a_{2}+b_{2}\right)=a_{1} a_{2}+a_{1} b_{2} \in\left(N:_{R} M\right)$ by Theorem 3. Since $a_{1} a_{2} \in\left(N:_{R} M\right)$ and $a_{1} a_{2}+a_{1} b_{2} \in\left(N:_{R} M\right)$, we conclude that $a_{1} b_{2} \in\left(N:_{R} M\right)$. Since $b_{1} K \subseteq M-G r(N)$ and $a_{1} K \nsubseteq M-G r(N)$, we conclude that $\left(a_{1}+b_{1}\right) K \nsubseteq M-G r(N)$. Since $\left(a_{1}+b_{1}\right) a_{2} K \subseteq N$ and neither $a_{2} K \subseteq M$ $G r(N)$ nor $\left(a_{1}+b_{1}\right) K \subseteq M-G r(N)$, we obtain that $\left(a_{1}+b_{1}\right) a_{2}=a_{1} a_{2}+b_{1} a_{2} \in$ $\left(N:_{R} M\right)$ by Theorem 3. Since $a_{1} a_{2} \in\left(N:_{R} M\right)$ and $a_{1} a_{2}+b_{1} a_{2} \in\left(N:_{R}\right.$ $M)$, we have $b_{1} a_{2} \in\left(N:_{R} M\right)$. Now, since $\left(a_{1}+b_{1}\right)\left(a_{2}+b_{2}\right) K \subseteq N$ and neither $\left(a_{1}+b_{1}\right) K \subseteq M-G r(N)$ nor $\left(a_{2}+b_{2}\right) K \subseteq M-G r(N)$, we conclude that $\left(a_{1}+b_{1}\right)\left(a_{2}+b_{2}\right)=a_{1} a_{2}+a_{1} b_{2}+b_{1} a_{2}+b_{1} b_{2} \in\left(N:_{R} M\right)$ by Theorem 3. Since $a_{1} a_{2}, a_{1} b_{2}, b_{1} a_{2} \in\left(N:_{R} M\right)$, we get $b_{1} b_{2} \in\left(N:_{R} M\right)$, a contradiction. Consequently $I_{1} K \subseteq M-G r(N)$ or $I_{2} K \subseteq M-G r(N)$, so we are done. 
Let $R$ be a graded ring and $S \subseteq h(R)$ be a multiplicatively closed subset of $R$. Then the ring of fraction $S^{-1} R$ is a graded ring. Indeed, $S^{-1} R=$ $\oplus_{g \in G}\left(S^{-1} R\right)_{g}$ where $\left(S^{-1} R\right)_{g}=\left\{\frac{r}{s}: r \in R, s \in S\right.$ and $\left.g=(\operatorname{deg} s)^{-1}(\operatorname{deg} r)\right\}$. Let $M$ be a graded module over a graded $\operatorname{ring} R$ and $S \subseteq h(R)$ be a multiplicatively closed subset of $R$. Then $S^{-1} M$ over a graded $\operatorname{ring} S^{-1} R$ is a graded module if $S^{-1} M=\oplus_{g \in G}\left(S^{-1} M\right)_{g}$ where $\left(S^{-1} M\right)_{g}=\left\{\frac{m}{s}: m \in M, s \in S\right.$ and $\left.g=(\operatorname{deg} s)^{-1}(\operatorname{deg} m)\right\}$. Here $h\left(S^{-1} R\right)=\cup_{g \in G}\left(S^{-1} R\right)_{g}$ and $h\left(S^{-1} M\right)=$ $\cup_{g \in G}\left(S^{-1} M\right)_{g}$.

Theorem 14. Let $R$ be a graded ring and $S \subseteq h(R)$ be a multiplicatively closed subset of $R$. If $N$ is a graded 2-absorbing primary submodule of $M$ and $S^{-1} N \neq S^{-1} M$, then $S^{-1} N$ is a graded 2-absorbing primary submodule of $S^{-1} M$.

Proof. Let $\frac{a}{s_{1}}, \frac{b}{s_{2}} \in h\left(S^{-1} R\right)$ and $\frac{m}{s_{3}} \in h\left(S^{-1} M\right)$ such that $\frac{a}{s_{1}} \frac{b}{s_{2}} \frac{m}{s_{3}} \in S^{-1} N$. Then there exists $u \in S$ with uabm $\in N$. This implies that $a b \in(N: R M)$ or uam $\in M-G r(N)$ or $u b m \in M-G r(N)$. If $a b \in\left(N:_{R} M\right)$, then $\frac{a}{s_{1}} \frac{b}{s_{2}}=\frac{a b}{s_{1} s_{2}} \in$ $S^{-1}\left(N:_{R} M\right) \subseteq\left(S^{-1} N:_{S^{-1}} S^{-1} M\right)$. Suppose that uam $\in M-G r(N)$. Then $\frac{a}{s_{1}} \frac{m}{s}=\frac{u a m}{u s_{1} s} \in S^{-1}(M-G r(N)) \subseteq\left(S^{-1} M\right)-\operatorname{Gr}\left(S^{-1} N\right)$. Similarly, if ubm $\in M$ $\operatorname{Gr}(N)$ then we have $\frac{b}{s_{2}} \frac{m}{s}=\frac{u b m}{u s_{2} s} \in\left(S^{-1} M\right)-\operatorname{Gr}\left(S^{-1} N\right)$.

Let $M$ and $M^{\prime}$ be graded modules over a $G$-graded ring $R$. A module homomorphism $f: M \rightarrow M^{\prime}$ is said to be graded if $f\left(M_{g}\right) \subseteq M_{g}^{\prime}$. $f$ is a graded isomorphism if it is a graded module homomorphism and an isomorphism.

Lemma 15. ([10, Corollary 1.3]) Let $M$ and $M^{\prime}$ be graded multiplication $R$-modules with $f: M \rightarrow M^{\prime}$ an graded $R$-module epimorphism. If $N$ is a graded submodule of $M$ containing $\operatorname{Ker}(f)$, then $f(M-G r(N))=M^{\prime}$ $\operatorname{Gr}(f(N))$.

Theorem 16. Let $M$ and $M^{\prime}$ be graded multiplication $R$-modules and $f: M \rightarrow M^{\prime}$ be a graded $R$-module homomorphism.

1. If $N^{\prime}$ is a graded 2-absorbing primary submodule of $M^{\prime}$, then $f^{-1}\left(N^{\prime}\right)$ is a graded 2-absorbing primary submodule of $M$.

2. If $f$ is epimorphism and $N$ is a graded 2-absorbing primary submodule of $M$ containing $\operatorname{Ker}(f)$, then $f(N)$ is a graded 2-absorbing primary submodule of $M^{\prime}$.

Proof. (1) Let $a, b \in h(R)$ and $m \in h(M)$ such that $a b m \in f^{-1}\left(N^{\prime}\right)$. Then $a b f(m) \in N^{\prime}$. Hence $a b \in\left(N^{\prime}: R M^{\prime}\right)$ or $a f(m) \in M^{\prime}-\operatorname{Gr}\left(N^{\prime}\right)$ or $b f(m) \in M^{\prime}$ $\operatorname{Gr}\left(N^{\prime}\right)$, and thus $a b \in\left(f^{-1}\left(N^{\prime}\right):_{R} M\right)$ or $a m \in f^{-1}\left(M^{\prime}-\operatorname{Gr}\left(N^{\prime}\right)\right)$ or $b m \in$ 
$f^{-1}\left(M^{\prime}-\operatorname{Gr}\left(N^{\prime}\right)\right)$. By using the inclusion $f^{-1}\left(M^{\prime}-\operatorname{Gr}\left(N^{\prime}\right)\right) \subseteq M-\operatorname{Gr}\left(f^{-1}\left(N^{\prime}\right)\right)$, we conclude that $f^{-1}\left(N^{\prime}\right)$ is a graded 2-absorbing primary submodule of $M$. (2) Let $a, b \in h(R), m^{\prime} \in h\left(M^{\prime}\right)$ and $a b m^{\prime} \in f(N)$. Since $f$ is epimorphism there exists $m \in h(M)$ such that $m^{\prime}=f(m)$ and so $f(a b m) \in f(N)$. Since $\operatorname{Ker}(f) \subseteq N$, we have $a b m \in N$. It implies that $a b \in(N: R M)$ or $a m \in M$ $G r(N)$ or $b m \in M-G r(N)$. Hence $a b \in\left(f(N):_{R} M^{\prime}\right)$ or $a m^{\prime} \in f(M-G r(N))=$ $M^{\prime}-\operatorname{Gr}(f(N))$ or $b m^{\prime} \in f(M-G r(N))=M^{\prime}-\operatorname{Gr}(f(N))$. Consequently $f(N)$ is a graded 2-absorbing primary submodule of $M^{\prime}$.

As an immediate consequence of Theorem 16 (2) we have the following Corollary.

Corollary 17. Let $M$ be a graded multiplication $R$-module and $L \subseteq N$ be graded submodules of $M$. If $N$ is a graded 2-absorbing primary submodule of $M$, then $N / L$ is a graded 2-absorbing primary submodule of $M / L$.

Let $R_{i}$ be a graded commutative ring with identity and $M_{i}$ be a graded $R_{i}$-module, for $i=1,2$. Let $R=R_{1} \times R_{2}$. Then $M=M_{1} \times M_{2}$ is a graded $R$-module and each graded submodule of $M$ is of the form $N=N_{1} \times N_{2}$ for some graded submodules $N_{1}$ of $M_{1}$ and $N_{2}$ of $M_{2}$. In addition, if $M_{i}$ is a graded multiplication $R_{i}$-module, for $i=1,2$, then $M$ is a graded multiplication $R$ module. In this case, for each graded submodule $N=N_{1} \times N_{2}$ of $M$ we have $M-G r(N)=M_{1}-\operatorname{Gr}\left(N_{1}\right) \times M_{2}-\operatorname{Gr}\left(N_{2}\right)$.

Theorem 18. Let $R=R_{1} \times R_{2}$ and $M=M_{1} \times M_{2}$ where $M_{1}$ is a graded multiplication $R_{1}$-module and $M_{2}$ is a graded multiplication $R_{2}$-module.

1. A proper graded submodule $K_{1}$ of $M_{1}$ is a graded 2-absorbing primary submodule if and only if $N=K_{1} \times M_{2}$ is a graded 2-absorbing primary submodule of $M$.

2. A proper graded submodule $K_{2}$ of $M_{2}$ is a graded 2-absorbing primary submodule if and only if $N=M_{1} \times K_{2}$ is a graded 2-absorbing primary submodule of $M$.

3. Assume that $M_{1}$ is a graded cyclic $R_{1}$-module and $M_{2}$ is a graded cyclic $R_{2}$-module. If $K_{1}$ and $K_{2}$ are graded primary submodules of $M_{1}$ and $M_{2}$, respectively, then $N=K_{1} \times K_{2}$ is a graded 2-absorbing primary submodule of $M$.

Proof. (1) Suppose that $N=K_{1} \times M_{2}$ is a graded 2-absorbing primary submodule of $M$. From our hypothesis, $N$ is proper, so $K_{1} \neq M_{1}$. Set $M^{\prime}=$ $\frac{M}{\{0\} \times M_{2}}$. Hence $N^{\prime}=\frac{N}{\{0\} \times M_{2}}$ is a graded 2-absorbing primary submodule of 
$M^{\prime}$ by Corollary 17. Also observe that $M^{\prime} \cong M_{1}$ and $N^{\prime} \cong K_{1}$. Thus $K_{1}$ is a graded 2-absorbing primary submodule of $M_{1}$. Conversely, if $K_{1}$ is a graded 2-absorbing primary submodule of $M_{1}$, then it is clear that $N=K_{1} \times M_{2}$ is a graded 2-absorbing primary submodule of $M$.

(2) It can be easily verified similar to (1).

(3) Assume that $N=K_{1} \times K_{2}$ where $K_{1}$ and $K_{2}$ are graded primary submodules of $M_{1}$ and $M_{2}$, respectively. Hence $\left(K_{1} \times M_{2}\right) \cap\left(M_{1} \times K_{2}\right)=K_{1} \times K_{2}=N$ is a graded 2-absorbing primary submodule of $M$, by parts (1) and (2) and Theorem 10.

\section{References}

[1] K. Al-Zoubi, R. Abu-Dawwa, On graded 2-absorbing and weakly graded 2-absorbing submodules, Journal of Mathematical Sciences: Advances and Applications, 28 (2014), 45-60.

[2] S. E. Atani, On graded weakly prime ideals, Turk J. Math., 30 (2006) 351-358.

[3] S. E. Atani, On graded prime submodules, Chiang Mai J. Sci., 33, No. 1 (2006) 3-7.

[4] S. E. Atani, On graded weakly prime submodules, Int. Math. Forum, 1, No. 2 (2006) 61-66.

[5] S. E. Atani and F. Farzalipour, Notes on the graded prime submodules, Int. Math. Forum, 1, No. 38 (2006) 1871-1880.

[6] S. E. Atani and F. Farzalipour, On Graded Secondary Modules, Turk J Math, 31 (2007) 371-378.

[7] S. E. Atani, R.E. Atani, Graded multiplication modules and the graded ideal $\theta_{g}(M)$, Turk J Math, 35 (2011) 1- 9.

[8] A. Badawi, U. Tekir and E. Yetkin, On 2-absorbing primary ideals over commutative rings, Bull. Korean Math. Soc., 51, No. 4 (2014), 1163-1173.

[9] Z.A. El-Bast and P.F. Smith, Multiplication modules, Comm. Algebra, 16 (1988) 755-779.

[10] R.L. McCasland and M.E. Moore, Radicals of submodules, Comm. Algebra, 19 (1991) 1327-1341.

[11] C. Nastasescu, F. Van Oystaeyen, Graded Ring Theory, Amsterdam, Holland 28 (1982).

[12] K. H Oral, U. Tekir and A. G. Agargun, On graded prime and primary submodules, Turk. J. Math., 35 (2011), 159-167.

[13] M. Refai, K. Al-Zoubi, On graded primary ideals, Turk J. Math., 28 (2004), 217-229.

[14] F. Van Oystaeyen, J. P. Van Deuren, Arithmetically graded rings, Lecture Notes in Math., Ring Theory, Proceedings 825 (1980), 279-284.

[15] E. Yetkin Celikel, On Graded 2-absorbing primary ideals, Submitted. 
